\title{
IAU SYMPOSIUM 156 RESOLUTIONS
}

\section{RESOLUTION 1. HISTORICAL ASTROMETRIC OBSERVATIONS FOR EARTH ORIENTATION DATA}

\section{IAU SYMPOSIUM 156 RECOGNIZING:}

1. the danger that valuable original astrometric observations might be lost, if not collected soon, and

2. the importance for further geodynamics studies, of the new reduction of Earth orientation parameters in the Hipparcos reference system, since the beginning of the century,

\section{URGES:}

the observatories selected by the IAU Commission 19 working group to convert their results of latitude, universal time, and zenith distance, based on individual star observations, into machine readable form and make them available to the WG at their earliest convenience, together with a complete description of the standards used for their reduction.

\section{RESOLUTION 2. ASTROMETRIC OBSERVATIONS}

\section{IAU SYMPOSIUM 156 RECOGNIZING:}

1. the importance to evaluate, as seismic precursors, the possible occurrence of local vertical variations in active areas, and

2. that an IAG-Special Study Group is studying this problem,

\section{RECOMMENDS THAT:}

1. past and present optical time and latitude observations be made available to the analysis center at Shanghai Observatory,

2. the necessary support be given to the concerned observatories and stations and to

$$
\text { xxix }
$$


Shanghai Observatory.

\section{RESOLUTION 3. VERY LONG BASELINE INTERFEROMETRY (VLBI)}

\section{IAU SYMPOSIUM 156 RECOGNIZING THAT:}

1. VLBI provides high accuracy, long-term reference frames, orientation, and connections, and

2. there is a need to maintain this reference frame and determine the relationship between it and the optical sources.

AND NOTING;

1. the QUASAR system has been delayed by one year,

2. the recent loss of one, and possibly two radio telescopes, in the USA due to natural disasters and the need to rebuild those telescopes, and

3. that VLBI observatories require continuing operational resources in order to maintain these high accuracy reference frames and to determine the Earth orientation parameters,

\section{URGES:}

all national agencies charged with the operation of VLBI and optical observatories to provide the resources required to:

1. develop and maintain networks of VLBI observatories spread around the world,

2. continue the observations necessary to improve the accuracy of the current reference system and to determine Earth Orientation Parameters, and

3. encourage and schedule the necessary VLBI and optical observations to determine and maintain the relationships between the radio and optical reference frames.

\section{RESOLUTION 4. MILLI AND MICRO ARCSECOND ASTROMETRY}

\section{IAU SYMPOSIUM 156 RECOGNIZING:}

1. that significant improvements in astrometric accuracies can be achieved by observations from above the Earth's atmosphere,

2. the impact on astronomy, astrophysics, and geodynamics from astrometry with 
milliarcsecond accuracy, and

3. that astrometric observations at the micro-arcseconds level provide data of great interest to astrophysics, geodynamics, cosmology, and extra solar system planetary investigations,

\section{AND NOTING THAT:}

the space agencies are studying both satellite and lunar based optical and radio observing programs,

\section{URGES THAT:}

1. efforts are supported and further initiatives taken to achieve micro arcseconds accuracies in optical and radio wavelengths for large numbers of sources,

2. the best accuracy on a cost effective basis be pursued,

3. international cooperation to achieve space based astrometry be encouraged,

4. and an IAU symposium be held at the IAU General Assembly 1994 with the title "Astronomy and Astrophysics from Milli and Micro Arcseconds Astrometry" to facilitate the above purpose.

\section{RESOLUTION 5. APPRECIATION}

\section{IAU SYMPOSIUM 156 CONSIDERING THAT:}

1. the symposium was an informative, interesting, and enjoyable meeting held in Shanghai, China,

2. the meeting achieved the desired goal of an interdisciplinary exchange of scientific knowledge and future requirements,

\section{AND NOTING}

the impressive recent progress that has been made in instrumentation, operations, research, and knowledge in astronomy and geodynamics in China,

\section{EXTENDS}

our appreciation and thanks to the Shanghai Astronomical Observatory of the Chinese Academy of Sciences and all the members of the Local Organizing Committee for their contribution to making the meeting an outstanding success. 